\title{
The diameter and evolutionary state of Procyon A
}

\section{Multi-technique modeling using asteroseismic and interferometric constraints}

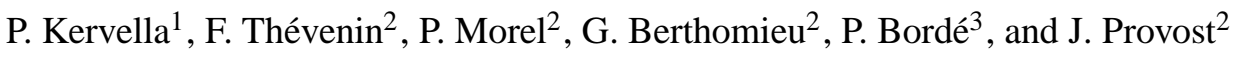 \\ 1 European Southern Observatory, Casilla 19001, Vitacura, Santiago 19, Chile \\ 2 Département Cassini, UMR CNRS 6529, Observatoire de la Côte d'Azur, BP 4229, 06304 Nice Cedex 4, France \\ 3 LESIA, Observatoire de Paris-Meudon, 5, place Jules Janssen, 92195 Meudon Cedex, France
}

Received 19 May 2003 / Accepted 20 August 2003

\begin{abstract}
We report the angular diameter measurement obtained with the VINCI/VLTI instrument on the nearby star Procyon A $\left(\alpha \mathrm{CMi}\right.$ A, F5IV-V), at a relative precision of $\pm 0.9 \%$. We obtain a uniform disk angular diameter in the $K$ band of $\theta_{\mathrm{UD}}=$ $5.376 \pm 0.047$ mas and a limb darkened value of $\theta_{\mathrm{LD}}=5.448 \pm 0.053$ mas. Together with the HIPPARCos parallax, this gives a linear diameter of $2.048 \pm 0.025 D_{\odot}$. We use this result in combination with spectroscopic, photometric and asteroseismic constraints to model this star with the CESAM code. One set of modeling parameters that reproduces the observations within their error bars are an age of $2314 \mathrm{Myr}$, an initial helium mass fraction $Y_{\mathrm{i}}=0.301$ and an initial mass ratio of heavy elements to hydrogen $\left(\frac{Z}{X}\right)_{\mathrm{i}}=0.0314$. We also computed the adiabatic oscillation spectrum of our model of Procyon A, giving a mean large frequency separation of $\overline{\Delta v_{0}} \approx 54.7 \mu \mathrm{Hz}$. This value is in agreement with the seismic observations by Martić et al. (1999, 2001). The interferometric diameter and the asteroseismic large frequency spacing together suggest a mass closer to $1.4 M_{\odot}$ than to 1.5 $M_{\odot}$. We conclude that Procyon is currently ending its life on the main sequence, as its luminosity class indicates.
\end{abstract}

Key words. stars: individual: $\alpha \mathrm{CMi}$ - stars: fundamental parameters - stars: evolution - techniques: interferometric

\section{Introduction}

Procyon A ( $\alpha \mathrm{CMi}$, HD 61421, HIP 37279) is among the brightest stars in the sky and is easily visible to the naked eye. This made it an ideal target for a number of spectrophotometric calibration works. It is also a visual binary star (ADS 6251A) classified F5 IV-V, with a white dwarf (WD) companion orbiting the main component in 40 years. The influence of this massive companion on the apparent motion of Procyon was discovered by Bessel (1844). Girard et al. (2000) have measured precisely the orbit of the pair, and obtained masses of $1.497 \pm 0.037 M_{\odot}$ and $0.602 \pm 0.015 M_{\odot}$, respectively for Procyon A and B. It has also been an asteroseismic target since a decade and Martic et al. (1999 and 2001 hereafter M99 and M01) have measured a large frequency spacing of respectively 55 and $54 \mu \mathrm{Hz}$. These asteroseismic observations provide strong constraints on stellar interior models, and on macroscopic stellar parameters like mass and radius. Comparing the direct interferometric measurements of this star to its model diameter is therefore important to cross-validate both approaches.

Matching Procyon's position in the HR diagram has been recognized as of great difficulty by Guenther \& Demarque (1993, hereafter GD93). The reason is the poorly known mass

Send offprint requests to: P. Kervella,

e-mail: Pierre.Kervella@eso.org and metallicity $Z$ of the star. We note that among the computed models only their model $b$ is close to the mean large frequency spacing measured by M99-M01. Because numerous new studies and observational constraints (like the direct diameter) exist today, we re-examine the status of Procyon A in helium content $Y$ and in age. We first review and present the adopted fundamental parameters of Procyon A in Sect.2, part of them being used for the limb-darkening in Sect. 3, where we present our new interferometric observations and the associated data processing. In Sect. 4, we detail several models of Procyon computed with the CESAM code (Morel 1997). These models are constrained using the spectroscopic effective temperature and the linear diameter value that we derived from the VINCI/VLTI observations. Thus, we avoid to fit the luminosity for which bolometric corrections are rather uncertain for stellar luminosity class IV-V. A study of the asteroseismic frequencies is then presented in Sect. 5, before the conclusion.

\section{Fundamental parameters}

Physical properties of Procyon A are well known thanks to the carefully measured orbit of the system and accurate HIPPARcos parallax. Its mass has been recently measured by Girard et al. (2000) at $M_{\mathrm{A}}=1.497 \pm 0.037 M_{\odot}$, adopting a parallax of 
$283.2 \pm 1.5$ mas. This mass is computed using a large number of observations of inhomogeneous quality. The same authors, when they limit their observation sample to the excellent images obtained with the WFPC2, found $M_{\mathrm{A}}=1.465 \pm 0.041 M_{\odot}$. If we replace the parallax used by Girard et al. (2000) by the parallax from the HIPPARCos catalogue, $285.93 \pm 0.88$ mas, the sum of the masses of the binary decreases by $2.9 \%$. Consequently, masses become respectively $M_{\mathrm{A}}=1.42 \pm$ $0.04 M_{\odot}$ for Procyon A and $M_{\mathrm{B}}=0.575 \pm 0.017 M_{\odot}$ for the white dwarf Procyon B. Allende Prieto et al. (2002, hereafter AP02), using the HIPPARCOS parallax of Procyon A, have estimated its mass to be $1.42 \pm 0.06 M_{\odot}$. From these works, we can conclude that the mass of Procyon is probably between 1.4 and $1.5 M_{\odot}$. Consequently, we investigate these two values in our study. The other adopted stellar parameters are summarized in Table 1.

The bolometric luminosity is given by Steffen (1985) $\log \left(L_{\star} / L_{\odot}\right)=0.85 \pm 0.06$. The photospheric properties of Procyon have been carefully studied by AP02, who derived an effective temperature $T_{\text {eff }}=6512 \mathrm{~K}$. This value is based on the angular diameter measured in the visible by the Mark III optical interferometer (Mozurkewich et al. 1991). Detailed 3D stellar atmosphere simulations have led AP02 to correct this value of $T_{\text {eff }}$ by $80 \mathrm{~K}$. Following their results, we adopt $T_{\text {eff }}=$ $6530 \pm 50 \mathrm{~K}$ in our computations with a surface gravity of $\log g=3.96 \pm 0.02$. It is interesting to notice that over the last twenty years, the effective temperature estimates for Procyon are spread between 6545 and $6811 \mathrm{~K}$ (Cayrel et al. 1997). Adopting a color index of $B-V=0.421$ and using the calibration by Alonso (1996) we find $T_{\text {eff }}=6551 \mathrm{~K}$ which is very close to the value proposed by AP02.

The iron abundance at surface of $[\mathrm{Fe} / \mathrm{H}]=-0.05 \pm$ 0.03 dex with respect to the solar one is also taken from AP02. Therefore we adopt for the modeling of the internal structure of Procyon A a chemical mixture which is calculated from the iron abundance using the approximation:

$$
\begin{aligned}
{[\mathrm{Fe} / \mathrm{H}]_{\mathrm{s}} } & \equiv \log \left(\frac{Z_{\mathrm{Fe}}}{Z}\right)_{\mathrm{s}}+\log \left(\frac{Z}{X}\right)_{\mathrm{s}}-\log \left(\frac{Z_{\mathrm{Fe}}}{X}\right)_{\odot} \\
& \simeq \log \left(\frac{Z}{X}\right)_{\mathrm{s}}-\log \left(\frac{Z}{X}\right)_{\odot}
\end{aligned}
$$

where $\left(\frac{Z_{\mathrm{Fe}}}{Z}\right)_{\mathrm{s}}$ is the iron mass fraction within $Z$. We use the solar mixture of Grevesse \& Noels (1993) then $\left(\frac{Z}{X}\right)_{\odot}=0.0245$. As we shall see in Sect. 4 , we adopt at the age of Procyon A a surface abundance $\left(\frac{Z}{X}\right)_{\mathrm{S}}=0.0217 \pm 0.0017$. Note that this adopted error bar of AP02 is very small. Because the white dwarf (WD) Procyon B has already experienced the AGB phase, it is not excluded that material coming from this star can have contaminated the narrow surface convection layer of Procyon A. However, there is no evidence of such an effect in the published table of abundances of Procyon A (Steffen 1985). Enriched elements like "s" or other elements coming from Procyon B during its post-AGB phase do not seem to have migrated as gas or dust in the atmosphere of Procyon A. The mass transfer during this short period of the WD progenitor life could not exceed the accretion of the wind passing close to the star. Therefore,
Table 1. Relevant parameters of $\alpha \mathrm{CMi}$ (Procyon) and its calibrator $\alpha \mathrm{CMa}$ (Sirius). For Sirius, see also Kervella et al. (2003d).

\begin{tabular}{lcc}
\hline \hline & $\alpha$ CMi & $\alpha$ CMa \\
& HD 61421 & HD 48915 \\
\hline$m_{V}$ & 0.34 & -1.47 \\
$m_{K}$ & -0.65 & -1.31 \\
Spectral Type & F5IV-V & A1Vm \\
$M\left(M_{\odot}\right)$ & $1.42 \pm 0.04$ & $2.12 \pm 0.02$ \\
$T_{\text {eff }}(\mathrm{K})$ & $6530 \pm 50$ & $9900 \pm 200$ \\
$\log g$ & 3.96 & 4.30 \\
{$[\mathrm{Fe} / \mathrm{H}]$} & $-0.05 \pm 0.03$ & $0.50 \pm 0.20$ \\
$v \sin i$ & $3.16 \pm 0.50$ & $16.0 \pm 1.0$ \\
$\pi^{a}(\mathrm{mas})$ & $285.93 \pm 0.88$ & $379.22 \pm 1.58$ \\
$\theta_{\mathrm{UD}}(\mathrm{mas})$ & $\mathbf{5 . 3 7 6} \pm \mathbf{0 . 0 4 7}$ & $5.94 \pm 0.02^{b}$ \\
$\theta_{\mathrm{LD}}(\mathrm{mas})$ & $\mathbf{5 . 4 4 8} \pm \mathbf{0 . 0 5 3}$ & $6.04 \pm 0.02^{b}$ \\
\hline
\end{tabular}

a Parallax values from HIPPARCos (Perryman et al. 1997).

${ }^{b}$ Sirius $\theta_{\mathrm{UD}}$ and $\theta_{\mathrm{LD}}$ are taken from Kervella et al. (2003d).

except if the two stars have filled their Roche lobe, the mass of Procyon A has only been changed by a negligible amount.

The projected rotational velocity of Procyon A has been determined by many authors who derived values smaller than $5.0 \mathrm{~km} \mathrm{~s}^{-1}$. Considering in particular the value proposed by AP02, $v \sin i=3.2 \pm 0.5 \mathrm{~km} \mathrm{~s}^{-1}$, we conclude that Procyon A is a slow rotator and we neglect the rotational velocity in our modeling of its internal structure. The semi-major axis of the Procyon orbit being $\alpha \simeq 4$ ".5 (Girard et al. 2000), the distance between the two companions amounts to $\simeq 16 \mathrm{AU}$. The tidal interaction between the two components is therefore negligible.

\section{Interferometric angular diameter}

\subsection{Instrumental setup and data processing}

The European Southern Observatory's Very Large Telescope Interferometer (Glindemann et al. 2000) is operated on top of the Cerro Paranal, in Northern Chile, since March 2001. The observations reported here were done with two test siderostats (0.35 $\mathrm{m}$ aperture) and the VINCI beam combiner (Kervella et al. 2000, 2003a). The Procyon visibility measurements were all obtained on the B3-D3 baseline, $24 \mathrm{~m}$ in ground length. We used a regular $K$ band filter $(\lambda=2.0-2.4 \mu \mathrm{m})$ for these observations. The effective wavelength changes slightly depending on the spectral type of the observed target. For Procyon, we estimate $\lambda_{\text {eff }}=2.178 \pm 0.003 \mu \mathrm{m}$. This error bar adds quadratically a relative systematic uncertainty of $0.15 \%$ on the final limb darkened angular diameter error bar.

The raw data processing has been achieved using an improved version of the standard data reduction pipeline (Kervella et al. 2003c), whose general principle is similar to the original FLUOR algorithm (Coudé du Foresto et al. 1997). The two calibrated output interferograms are subtracted to remove residual photometric fluctuations. Instead of the classical Fourier analysis, we implemented a wavelet based timefrequency analysis (Ségransan et al. 1999). The output of the 


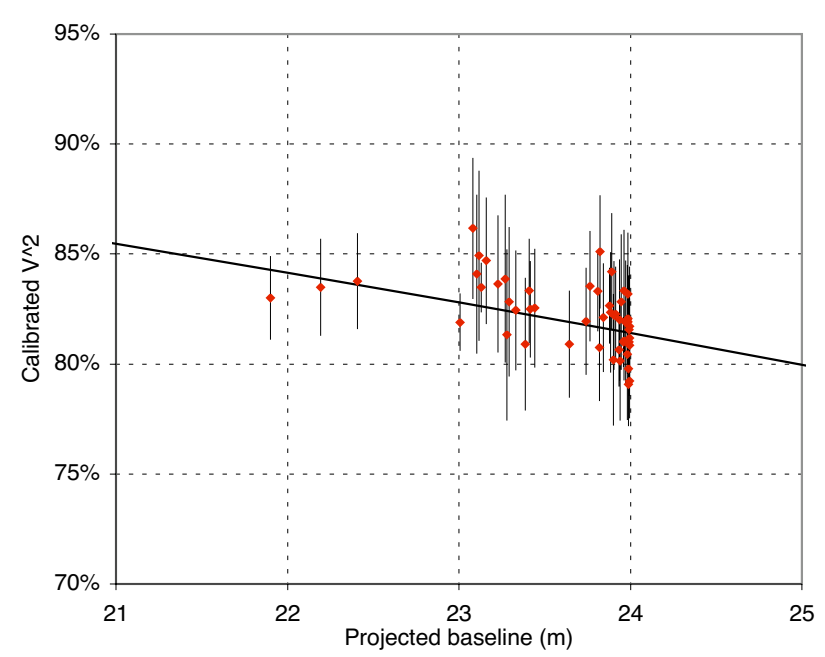

Fig. 1. Squared visibility measurements obtained on Procyon and best fit model (solid line).

processing pipeline is a single value of the squared coherence factor $\mu^{2}$ for each series of 500 interferograms and the bootstrapped error bar.

\subsection{Visibility calibration}

Sirius was chosen as the calibrator for Procyon observations as its limb darkened (LD) angular diameter has been measured recently with high precision by Kervella et al. (2003d), using VINCI and the $66 \mathrm{~m}$ E0-G1 baseline. Table 1 gives the relevant parameters of Procyon and Sirius. The interferometric efficiency (IE) of the instrument was estimated from the $\mu^{2}$ values obtained on Sirius a short time before and after Procyon (typically $15 \mathrm{~min}$ ). Thanks to the very high SNR of the Sirius interferograms, we were able to obtain very small relative errors on the IE values, typically $0.2 \%$ for a series of 500 interferograms. The error bar on the angular diameter of Sirius is treated as a systematic error in the calibration process, and is therefore not averaged for multiple observations. For the visibility fit, we took into account simultaneously the limb darkening and the bandwidth smearing, as described in Kervella et al. (2003b, 2003d).

Our February 2003 observation campaign of Procyon resulted in a total of 53 calibrated $V^{2}$ measurements, from 23256 processed interferograms. In the calibration process, we separated clearly the statistical and systematic error contributions. This is essential to avoid underestimating the final error bars of the fit. The latter corresponds to the uncertainty on the angular diameter of Sirius. In spite of the fact that the angular size of Sirius is larger than Procyon's, the small error on its measured value $( \pm 0.3 \%)$ translates into a small calibration error. As a consequence, the global error on the angular size of Procyon is dominated by the statistical contribution by a factor of two compared to the systematic calibration error: the uncertainty on the angular size of Sirius is not limiting our final precision. The visibility points as a function of the projected baseline are presented in Fig. 1, and their residual scattering around the best fit model in Fig. 2.

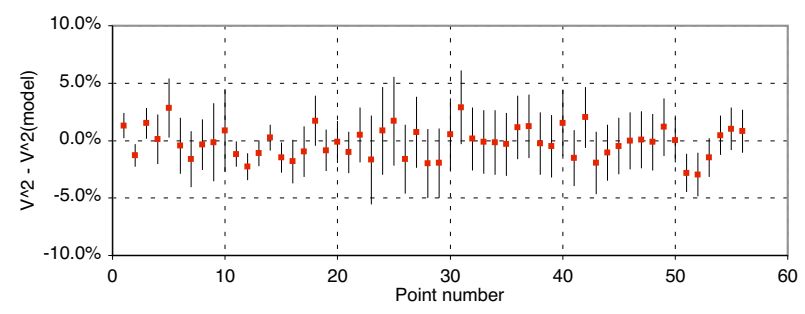

Fig. 2. Scattering of the Procyon measured visibilities around the best fit $V^{2}$ model. The plotted error bars are the quadratic sum of the statistical and systematic contributions.

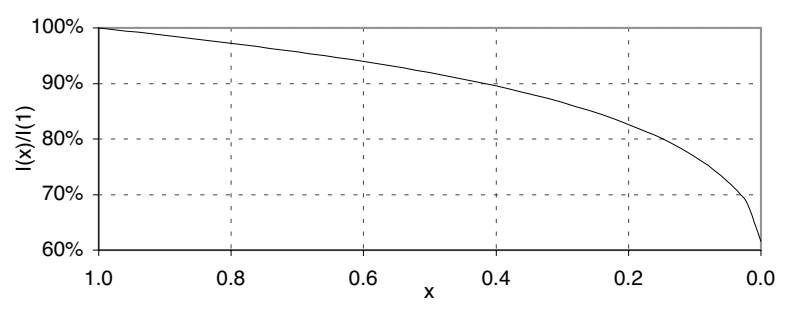

Fig. 3. Intensity profile of Procyon, from the four parameters law of Claret (2000).

\subsection{Limb darkened angular diameter and linear size}

The intensity profile $I(\mu) / I(1)$ that we chose for Procyon was computed by Claret et al. (2000), based on the ATLAS9 model atmospheres (Kurucz 1992). It is a four parameters law:

$I(x) / I(1)=1-\sum_{k=1}^{4} a_{k}\left(1-x^{\frac{k}{2}}\right)$

which coefficients are $a_{1}=0.5089, a_{2}=0.0822, a_{3}=$ -0.3978 , and $a_{4}=0.1967$. The parameter $x=\cos (\theta)$ is the cosine of the azimuth of a surface element of the star. The resulting profile is shown in Fig. 3. AP02 have identified an influence of the convective granulation of Procyon on its limb darkening at visible wavelengths. Though, the amplitude of this effect is already very small at $\lambda=1.0 \mu \mathrm{m}$, and we neglect it for our observations at $\lambda=2.2 \mu \mathrm{m}$.

We obtain directly the LD angular diameter of Procyon from a classical $\chi^{2}$ minimization, $\theta_{\mathrm{LD}}=5.448 \pm 0.053$ mas. Using a simple uniform disk model, we find a value of $\theta_{\mathrm{UD}}=$ $5.376 \pm 0.047$ mas.

We can compare the LD value with the previously published interferometric measurements, listed in Table 2. Our value of $\theta_{\mathrm{LD}}$ is compatible with previous measurements, that were all obtained using visible wavelength observations. It is also in excellent agreement with the average of all published values. From the VINCI/VLTI value of $\theta_{\mathrm{LD}}$ and the HIPPARcos parallax, we deduce a linear diameter of $2.048 \pm 0.025 D_{\odot}$. Computing the average of all published $\theta_{\mathrm{LD}}$ values, we obtain a linear radius of $R_{\star}=2.047 \pm 0.020 R_{\odot}$ for Procyon A, statistically identical to our result.

\section{Modeling}

The modeling of Procyon A is based on the matching of spectroscopic effective temperature, surface metallicity and 
Table 2. Interferometric measurements of the angular diameter of Procyon A from the literature.

\begin{tabular}{|c|c|c|c|}
\hline Instrument & $\lambda(\mu \mathrm{m})$ & $\theta_{\mathrm{UD}}(\mathrm{mas})$ & $\theta_{\mathrm{LD}}(\mathrm{mas})$ \\
\hline Intensity interf. ${ }^{a}$ & 0.45 & $5.10 \pm 0.16$ & $5.41 \pm 0.17$ \\
\hline Mark III ${ }^{b}$ & 0.80 & $5.26 \pm 0.05$ & \\
\hline Mark III $^{b}$ & 0.45 & $5.14 \pm 0.05$ & \\
\hline Mark III ${ }^{c}$ & 0.80 & & $5.46 \pm 0.08$ \\
\hline $\mathrm{NPOI}^{c}$ & 0.74 & $5.19 \pm 0.04$ & $5.43 \pm 0.07$ \\
\hline VINCI/VLTI & 2.18 & $5.38 \pm 0.05$ & $5.45 \pm 0.05$ \\
\hline Average value & & & $5.445 \pm 0.035$ \\
\hline
\end{tabular}

interferometric radius by a "satisfactory" evolutionary model for a given mass. The constraint between $R_{\star}$ and $T_{\text {eff }}$ within their error bars is illustrated in Fig. 4 by the hatched parallelogram, while the constraint between the $T_{\text {eff }}$ and $L_{\star}$ is illustrated by the dashed rectangle. That emphazises the advantage of the use of the measured $R_{\star}$ instead of the $L_{\star}$ which depends of photometric calibrations and bolometric corrections.

Table 3 shows the characteristic and initial modeling parameters of the star. The ordinary assumptions of stellar modeling are made, i.e. spherical symmetry, no rotation, no magnetic field and no mass loss. The relevant nuclear reaction rates are taken from the NACRE compilation by Angulo et al.(1999). The equation of state adopted is taken from Eggleton et al. (1973), and the opacities are from the OPAL database (Iglesias \& Rogers 1996), using the Grevesse \& Noels (1993) mixture. The microscopic diffusion is described using the formalism of Burgers (1969) with the resistance coefficients of Paquette et al. (1986). We take into account the radiative diffusivity as recommended by Morel \& Thévenin(2002), that limits the efficiency of the microscopic diffusion in outerlayers of stars with intermediate masses. The atmosphere is restored using Hopf's law (Mihalas 1978). The definition of the radius of the star is the bolometric radius, where $T\left(R_{\star}\right)=T_{\text {eff }}$. In the convection zones the temperature gradient is computed according to the MLT $_{\mathrm{CM}}$ convection theory with a mixing length parameter of $\Lambda=1$ (Canuto \& Mazzitelli 1991, 1992).

Following the discussion of Sect. 2 we investigate the sensitivity of our models to a variation of the mass of Procyon in the range 1.4 to $1.5 M_{\odot}$. We obtained a "satisfactory" model $a$ (Fig. 4), detailed in Table 3, which reaches the hatched parallelogram corresponding to an age of $2314 \mathrm{Myr}$. Its evolutionary state corresponds closely to the end of the main sequence, when the convective core has disappeared owing to the exhaustion of the hydrogen at center. We present in Fig. 5 the variation for model $a$ of $X_{\mathrm{s}}, Y_{\mathrm{s}}$ and of $[\mathrm{Fe} / \mathrm{H}]_{\mathrm{s}}$ as a function of the age. From the ZAMS until about $1 \mathrm{Gyr}$, due to the gravitational settling, the surface abundances of helium and heavy elements (resp. hydrogen) decrease (resp. increases). After $1 \mathrm{Gyr}$, the density in the envelope decreases due to the enlargement of radius. This leads to an increase of the radiative mixing, with the

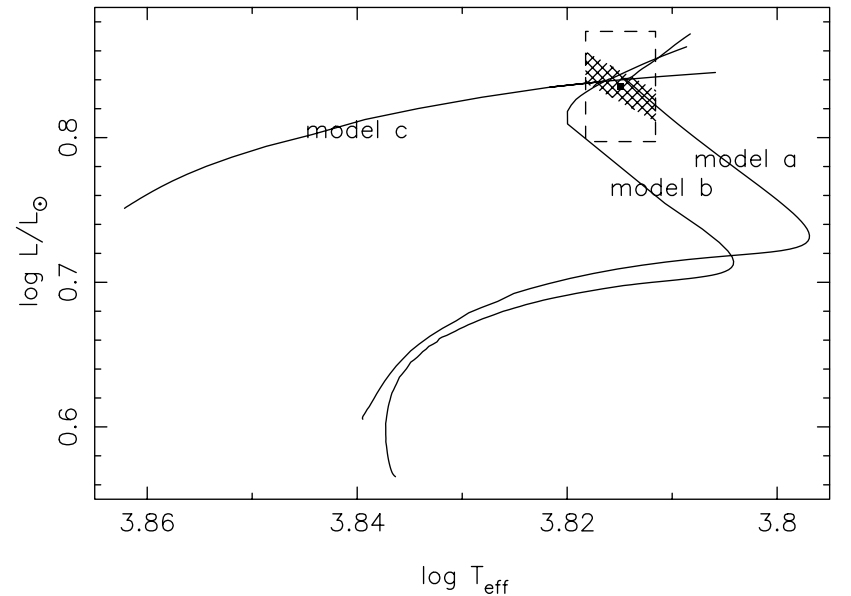

Fig. 4. Evolutionary tracks in the HR diagram of models of Procyon A (see Table 3). Dashed rectangle delimits the uncertainty domain for luminosity and effective temperature, while the hatched area delimits the uncertainty domain for effective temperature and the interferometric radius.

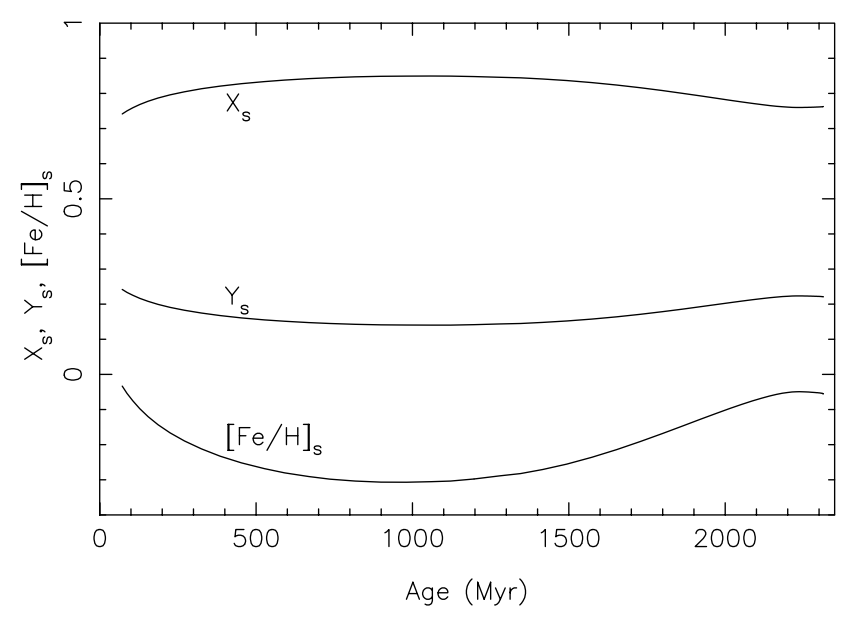

Fig. 5. Changes with respect to time for $X_{\mathrm{s}}, Y_{\mathrm{s}}$ and $[\mathrm{Fe} / \mathrm{H}]_{\mathrm{s}}$ for model $a$ of Procyon.

consequence of a dredge-up which increases (resp. decreases) $Y_{\mathrm{s}}$ and $[\mathrm{Fe} / \mathrm{H}]_{\mathrm{s}}$ (resp. $\left.X_{\mathrm{s}}\right)$ at the surface.

We also computed a "satisfactory" model $b$ without microscopic diffusion in order to estimate its effect on the age of the star and we found that suppressing the diffusion increases the age by $\sim 400$ Myr. The evolutionary state of this model is the beginning of $\mathrm{H}$ burning in a shell.

Following the prescriptions of Schaller et al.(1992) we have computed additional models that include overshooting of the convective core of radius $R_{\text {co }}$ over the distance $O_{\mathrm{v}}=$ $A \min \left(H_{\mathrm{p}}, R_{\mathrm{co}}\right)$. It has been impossible to find a "satisfactory" model reaching the narrow hatched area of the HR diagram with parameters $A>0.03$.

Finally, we computed a model $c$ with Girard et al. (2000) mass: $1.50 M_{\odot}$ and find an age of $1.3 \mathrm{Gyr}$ without overshoot. We also computed a model with an overshoot of 0.15 in agreement with Ribas et al. (2000) for stars of mass lower than $1.5 M_{\odot}$ resulting in a model which increases the age of the star to 1.55 Gyr. 
Table 3. Procyon A models (without overshoot, see text) lying within uncertainty box in the HR diagram. The subscripts " $i$ " and " " respectively refer to initial values and surface quantity at present day. "c" refers to the central value.

\begin{tabular}{lccc}
\hline \hline Model & $a$ & $b$ & $c$ \\
\hline$m / M_{\odot}$ & 1.42 & 1.42 & 1.50 \\
$Y_{\mathrm{i}}$ & 0.3012 & 0.2580 & 0.345 \\
$Y_{\mathrm{s}}$ & 0.2209 & 0.2580 & 0.202 \\
$\left(\frac{Z}{X}\right)_{\mathrm{i}}$ & 0.03140 & 0.0218 & 0.0450 \\
$\left(\frac{Z}{X}\right)_{\mathrm{s}}$ & 0.02157 & 0.0218 & 0.0220 \\
diffusion & yes & no & yes \\
$X_{\mathrm{c}}$ & 0.00051 & 0.00000 & 0.2180 \\
age $(\mathrm{Myr})$ & 2314 & 2710 & 1300 \\
$T_{\text {eff }}(\mathrm{K})$ & 6524 & 6547 & 6553 \\
$\log g$ & 3.960 & 3.967 & 3.994 \\
{$[\mathrm{Fe} / \mathrm{H}]_{\mathrm{i}}$} & +0.107 & -0.051 & +0.264 \\
{$[\mathrm{Fe} / \mathrm{H}]_{\mathrm{s}}$} & -0.055 & -0.051 & -0.043 \\
$\log \left(L / L_{\odot}\right)$ & 0.8409 & 0.8405 & 0.8390 \\
$R / R_{\odot}$ & 2.0649 & 2.0495 & 2.0420 \\
$\overline{\Delta v_{0}}(\mu \mathrm{Hz})$ & 54.7 & 55.4 & 56.4 \\
\hline
\end{tabular}

The parameters for all models are listed in Table 3. Figure 4 shows the corresponding evolutionary tracks in the HR diagram. We note that our models fit the value of $\log g=3.96$ proposed by AP02.

\section{Asteroseismology}

Asteroseismic observations of Procyon A have been reported by M99 and M01 and indicate an excess of power around 1 milliHertz with a probable frequency spacing of 54 to $55 \mu \mathrm{Hz}$.

The narrow convective envelope of the star we consider may stochastically excite solar-like oscillations. As in the Sun, these oscillations will have quasi equidistant frequencies at given degree.

For all the models, we have computed a set of adiabatic frequencies of the normal modes for degrees $\ell=0,1,2,3$. The set of frequencies of model $a$ are given in the frequency range 450 to $1600 \mu \mathrm{Hz}$ in Table 4 .

The large frequency spacing is defined as the difference between frequencies of modes with consecutive radial order $n$ : $\Delta v_{\ell}(n) \equiv v_{n, \ell}-v_{n-1, \ell}$. In the high frequency range, i.e. large radial orders, $\Delta v_{\ell}$ is almost constant with a mean value $\overline{\Delta v_{0}}$, strongly related to the mean density of the star. The computed frequencies are fitted to the following asymptotic relation (Berthomieu et al. 1993): $v_{n, \ell}=v_{0}+\overline{\Delta v_{\ell}}\left(n-n_{0}\right)+a_{\ell}\left(n-n_{0}\right)^{2}$. With $n_{0}=21, \ell=0$ and radial order $n$ between 17 and 26 we obtain an estimate of the mean large difference $\overline{\Delta v_{0}}$.

The mean large spacing of model $a$ is $54.7 \mu \mathrm{Hz}$ which fits well observational predictions of M99 \& M01 $\left(\overline{\Delta v_{0}} \sim\right.$ $54-55 \mu \mathrm{Hz}$ ). For the model $c$, the mean large spacing is found to be $56.4 \mu \mathrm{Hz}$, significantly different from the observed value. A mass as large as $1.5 M_{\odot}$ for Procyon A seems therefore improbable. Adopting the VINCI/VLTI radius, and looking at the scaling of Kjeldsen \& Bedding (2003),

$\overline{\Delta v_{\mathrm{osc}}} \sim 134.9 \sqrt{\frac{m / M_{\odot}}{\left(R_{\star} / R_{\odot}\right)^{3}}}(\mu \mathrm{Hz})$,
Table 4. Asteroseismic frequencies $(\mu \mathrm{Hz})$ of Procyon A for model $a$. The " $\star$ " correspond to " $g$ " modes which are presents in the frequency domain due to the evolutionary state of the star.

\begin{tabular}{ccccc}
\hline \hline$\ell=0$ & $\ell=1$ & $\ell=2$ & $\ell=3$ & $n$ \\
\hline 485.35 & 513.36 & 532.31 & 551.46 & 8 \\
- & - & - & 597.20 & $\star$ \\
537.93 & 563.43 & 585.90 & 605.61 & 9 \\
591.09 & 615.78 & 638.60 & 657.72 & 10 \\
643.23 & 666.79 & 689.80 & 708.98 & 11 \\
694.17 & 717.44 & 741.00 & 760.87 & 12 \\
745.32 & 769.15 & 793.75 & 814.22 & 13 \\
798.11 & 822.24 & 847.62 & 868.87 & 14 \\
- & - & 896.38 & - & $\star$ \\
852.14 & 876.76 & 904.48 & 923.72 & 15 \\
906.98 & 931.22 & 957.38 & 978.12 & 16 \\
961.42 & 985.26 & 1011.19 & 1031.92 & 17 \\
- & - & - & 1075.73 & $\star$ \\
1015.27 & 1038.85 & 1065.03 & 1086.34 & 18 \\
1069.16 & 1092.64 & 1119.00 & 1140.31 & 19 \\
1123.16 & 1146.76 & 1173.64 & 1195.27 & 20 \\
1177.86 & 1201.57 & 1228.79 & 1250.68 & 21 \\
1233.05 & 1256.82 & 1284.20 & 1306.18 & 22 \\
1288.46 & 1312.09 & 1339.65 & 1361.81 & 23 \\
1343.91 & 1367.55 & 1395.21 & 1417.43 & 24 \\
1399.42 & 1422.95 & 1450.65 & 1472.94 & 25 \\
1454.83 & 1478.24 & 1506.00 & 1528.39 & 26 \\
1510.12 & 1533.53 & 1561.40 & 1583.89 & 27 \\
\hline
\end{tabular}

the most straightforward way to decrease $\overline{\Delta v_{0}}$ is to decrease the mass of Procyon A.

Because the luminosity $L$ is in relation with $T_{\mathrm{eff}}$ and with the radius $R$ through the formula $L / L_{\odot}=\left(R / R_{\odot}\right)^{2}\left(T_{\text {eff }} / T_{\text {eff }}^{\odot}\right)^{4}$ we find from Eq. (3) the expression of the mean large spacing as a function of $L$ and $T_{\text {eff }}$,

$\overline{\Delta v_{\mathrm{osc}}} \sim 134.9 \frac{\sqrt{m / M_{\odot}}}{\left(L / L_{\odot}\right)^{3 / 4}\left(T / T_{\mathrm{eff}}\right)^{3}}(\mu \mathrm{Hz})$.

From this formula it is easy, if needed, to estimate the derivatives of the mean large spacing with respect to the mass, the effective temperature and the luminosity. To estimate the dependance between $\overline{\Delta v_{\mathrm{osc}}}$ and $Z_{\mathrm{i}}$ we have computed a modified model $a$ with $\Delta Z_{\mathrm{i}}=0.001$ and found a variation in the parallelogram error box of: $\Delta T_{\text {eff }}=22 \mathrm{~K}, \Delta L / L=0.008$, and $\Delta_{\text {age }}=33$ Myr. There were no significant changes with $Z_{\mathrm{i}}$ of $\overline{\Delta v_{\text {osc }}}$ as can be expected from Eq. (3).

\section{Discussion and conclusions}

We have reported in this paper our modelisation of Procyon A based on observations by long-baseline interferometry and asteroseismology. The use of the measured stellar diameter allows to reduce significantly the error bar on the luminosity of the star, as it does not require any bolometric corrections. This advantage has been demonstrated in this study and in the modeling of Sirius by Kervella et al. (2003d).

Our model shows that using the given set of parameters and given physics, Procyon A is currently finishing to burn its central hydrogen, and is at the phase where the convective core 
is disappearing. The error on the measured radius gives a narrow uncertainty of $10 \mathrm{Myr}$ on the deduced age. Provencal et al. (2002) have discussed the cooling time of the WD Procyon B and found that the progenitor ended its lifetime $1.7 \pm 0.1 \mathrm{Gyr}$ ago. We derive an age of 2314 Myr for Procyon A. Subtracting the cooling age of the WD companion to our determination of the age of Procyon A leads to a lifetime of $614 \mathrm{Myr}$ for the progenitor of Procyon $\mathrm{B}$. This indicates that the mass of the progenitor is approximately $2.5 M_{\odot}$. This value yields a mass of $\sim 0.57 M_{\odot}$ for the core of the corresponding ThermalPulsating-AGB star (for $Z=0.020$ ) (Bressan et al. 1993), which is the minimum possible value for the final mass of the WD (see also Jeffries 1997). This estimate of $0.57 M_{\odot}$ agrees very well with the mass of Procyon B that we deduced from Girard et al. (2000). We note that the age obtained with model $c$ is younger than the cooling age of Procyon B. This argument suggests a mass lower than $1.5 M_{\odot}$ for Procyon A and strengthens the asteroseismology results.

Further progress on the modeling of Procyon will be possible when the accuracy on the flux of the star is improved to less than $1 \%$. Waiting for such accuracy, the uniqueness of the solutions resulting from computed models fitting a narrow box in the HR diagram will come from future detailed asteroseismic studies. For example, other linear combinations of frequencies such as the small frequency spacings (see e.g. Gough 1991) will constrain the age and the mass of the star. We therefore encourage observers to progress on the asteroseismology of this star.

Large uncertainties also come from the adopted chemical abundance mixture $Z_{\mathrm{s}}$ which is still rather uncertain. Only a few chemical element abundances are measured today and most of them with a low accuracy. This uncertainty on $Z_{\mathrm{s}}$ is the largest source of error on the estimated initial helium content $Y_{\mathrm{i}}$ and on the age of Procyon. Thus, we recommend that surface abundances should be derived from 3D atmosphere studies, in particular for oxygen and other important donors of electrons.

Acknowledgements. The VLTI is operated by the European Southern Observatory at Cerro Paranal, Chile. The VINCI public commissioning data reported in this paper has been retrieved from the ESO/STECF Archive. The VINCI pipeline includes the wavelets processing technique, developed by D. Ségransan (Obs. de Genève). No VLTI observation would have been possible without the efforts of the ESO VLTI team, to whom we are grateful. This research has made use of the SIMBAD database at CDS, Strasbourg (France).

\section{References}

Allende Prieto, C., Asplund, M., Garcia López, R. J., \& Lambert, D. L. 2002, ApJ, 567, 544

Alonso, A., Arribas, S., \& Martínez-Roger, C. 1996, A\&A, 313, 873

Angulo, C., Arnould, M., Rayet, M., et al. 1999, Nucl. Phys. A, 656, 3

Berthomieu, G., Provost, J., Morel, P., \& Lebreton, Y. 1993, A\&A, 262,775

Bessel, F. W. 1844, MNRAS, 6, 136

Bressan, A., Fagotto, F., Bertelli, G., \& Chiosi, C. 1993, A\&AS, 100, 674

Burgers, J. M. 1969, Flow Equations for Composite Gases (New York: Academic Press)
Canuto, V. M., \& Mazitelli, I. 1991, ApJ, 370, 295

Canuto, V. M., \& Mazitelli, I. 1992, ApJ, 389, 729

Cayrel de Strobel, G., Soubiran, C., Friel, E. D., Ralite, N., \& Francois, P. 1997, A\&AS, 124, 299

Claret, A. 2000, A\&A, 363, 1081

Cohen, M., Walker, R. G., Barlow, M. J., \& Deacon, J. R. 1992, AJ, 104,1650

Cohen, M., Walker, R. G., Carter, B., et al. 1999, AJ, 117, 1864

Coudé du Foresto, V., Ridgway, S., \& Mariotti, J.-M. 1997, A\&AS, 121,379

Eggleton, P. P., Faulkner, J., \& Flannery, B. P. 1973, A\&A, 23, 325

Girard, T. M., Wu, H., Lee, J. T., et al. 2000, AJ, 119, 2428

Glindemann, A., Abuter, R., Carbognani, F., et al. 2000, SPIE, 4006, 2

Gough, D. O. 1991, in Comments on Helioseismic Inference, ed. Y. Osaki, \& H. Shibahashi, Progress of Seismology of the Sun and Stars (Springer Verlag, 283)

Grevesse, N., \& Noels, A. 1993, in Cosmic Abundances of the Elements, ed. N. Prantzos, E. Vangioni-Flam, \& M. Cassé, Origin and Evolution of the Elements (Cambridge University Press), 14

Guenther, D. B., \& Demarque, P. 1993, ApJ, 405, 298

Hanbury Brown, R., Davis, J., Lake, J. W., \& Thompson, R. J. 1974, MNRAS, 167, 475

Iglesias, C. A., \& Rogers, F. J. 1996, ApJ, 464, 943

Jeffries, R. D. 1997, MNRAS, 288, 585

Kervella, P., Coudé du Foresto, V., Glindemann, A., \& Hofmann, R. 2000, SPIE, 4006, 31

Kervella, P., Gitton, Ph., Ségransan, D., et al. 2003a, SPIE, 4838, 858

Kervella, P., Thévenin, F., Ségransan, D., et al. 2003b, A\&A, 404, 1087

Kervella, P., Ségransan, D., \& Coudé du Foresto, V. 2003c, in preparation

Kervella, P., Thévenin, F., Morel, P., et al. 2003d, A\&A, 408, 681

Kjeldsen, H., \& Bedding, T. 1995, A\&A, 293, 87

Kurucz, R. L. 1992, The Stellar Populations of Galaxies, IAU Symp., 149,225

Lemke, M. 1989, A\&A, 225, 125

Martic, M., Schmitt, J., Lebrun, J., et al. 1999, A\&A, 351, 993

Martic, M., Lebrun, J.-C., Schmit, J., Appourchaux, Th., \& Bertaux, J.-L. 2001, in SOHO 10 / GONG 2000 Workshop, ed. A. Wilson, ESA SP-464, 431

Mihalas, D. 1978, Stellar Atmospheres, 2nd ed. (San Francisco: W. H. Freeman and Co)

Mozurkewich, D., Johnston, K. J., Simon, R. S., et al. 1991, AJ, 101, 2207

Morel, P. 1997, A\&AS, 124, 597

Morel, P., \& Thévenin, F. 2002, A\&A, 390, 611

Nordgren, T. E., Sudol, J. J., \& Mozurkewich, D. 2001, AJ, 122. 2707

Paquette, C., Pelletier, C., Fontaine, G., \& Michaud, G. 1986, ApJS, 61,177

Provencal, J. L., Shipman, H. L., Koester, D., Wesemael, F., \& Bergeron, P. 2002, ApJ, 568, 324

Perryman, M. A. C., Lindegren, L., Kovalevsky, J., et al. 1997, The HIPPARcos Catalogue, A\&A, 323, 49

Ribas, I., Jordi, C., \& Giménez, À. 2000, MNRAS, 318, L55

Schaller, G., Schaerer, D., Meynet, G., \& Maeder, A. 1992, A\&AS, 96, 269

Ségransan, D., Forveille, T., Millan-Gabet, C. P. R., \& Traub, W. A. 1999, ASP Conf. Ser., 194, 290

Ségransan, D. 2001, Ph.D. Thesis, Grenoble

Steffen, M. 1985, A\&AS, 59, 403 